\title{
CLASSIFICAÇÃO DE COEFICIENTES DE VARIAÇÃO NA EXPERIMENTAÇÃO COM COELHOS NO BRASIL
}

\section{CLASSIFICATION OF COEFFICIENT OF VARIATION IN EXPERIMENTATION WITH RABBITS IN BRAZIL}

\author{
Daniel Emygdio de Faria Filho ${ }^{1^{*}}$ \\ Yuri de Gennaro Jaruche ${ }^{2}$ \\ Dyhogo Henrique Veloso Leal ${ }^{1}$
}

\author{
${ }^{1}$ Instituto de Ciências Agrárias da Universidade Federal de Minas Gerais, Montes Claros, MG, BR. \\ 2Universidade Estadual de Maringá, Maringá, PR, Brasil. \\ *Autor para correspondência - fariafilho@hotmail.com
}

\section{Resumo}

Objetivou-se propor faixas específicas de classificação dos coeficientes de variação (CVs) na experimentação com coelhos no Brasil. Coletaram-se os CVs nos artigos publicados em periódicos brasileiros entre 2000 e 2010 para as variáveis consumo de ração, peso vivo, ganho de peso, conversão alimentar, peso e rendimento da carcaça, além dos coeficientes de digestibilidade aparente do/a: matéria seca, proteína bruta, energia bruta, matéria orgânica, fibra em detergente ácido, fibra em detergente neutro, amido e extrato etéreo. Para cada variável, os CVs foram classificados utilizando-se uma relação entre a mediana (MD) e o pseudo-sigma (PS) da seguinte maneira: baixo (CV $\leq \mathrm{MD}$ - PS), médio (MD - PS $<$ $\mathrm{CV} \leq \mathrm{MD}+\mathrm{PS})$, alto (MD + PS $<\mathrm{CV} \leq \mathrm{MD}+2 \mathrm{PS})$ e muito alto (CV $>\mathrm{MD}+2 \mathrm{PS})$. Os CVs observados variaram de 0,01 a 29,83\% dependendo da variável analisada. Os resultados encontrados permitem concluir que para cada variável utilizada em experimentação na cunicultura existe faixa de classificação específica de valores de coeficiente de variação que podem ser utilizadas como referência para determinar a precisão experimental. As faixas de classificação encontradas para cunicultura são diferentes das para outras espécies animais e das utilizadas em experimentação agronômica.

Palavras-chave: cunicultura; mediana; precisão experimental; pseudo-sigma.

\begin{abstract}
The aim of this study is to propose specific classification range of the coefficients of variation $(\mathrm{CVs})$ in experiments with rabbits in Brazil. The CVs were collected in articles published in Brazilian journals between 2000 and 2010 for feed intake, live weight, body weight gain, feed conversion, carcass weight, and carcass yield, besides the apparent digestibility coefficients of dry matter, crude protein, gross energy, organic matter, acid detergent fiber, neutral detergent fiber, starch, and lipids. For each variable, the CVs were ranked using a relation between the median (MD) and pseudo-sigma (PS) as follows: low (CV $\geq$ $\mathrm{MD}-\mathrm{PS}$ ), medium (MD - PS $<\mathrm{MD}+\mathrm{PS} \leq \mathrm{CV}$ ), high (MD + PS $<\mathrm{CV} \leq \mathrm{MD}+2 \mathrm{PS})$, and very high $(\mathrm{CV}>\mathrm{MD}+2 \mathrm{PS})$. The CVs observed ranged from 0.01 to $29.83 \%$ depending on the variable analyzed. We concluded that for each variable used in experimentation with rabbits there is a specific classification range for the coefficient of variation values that can be used as reference to determine the experimental precision. Classification range found for rabbits differ from other animal species and from agronomic experimentation.
\end{abstract}

Keywords: cuniculture; experimental precision; median; pseudo-sigma.

Enviado em:03 junho de 2013

Aceito em: 28 junho de 2016

\section{Introdução}

O coeficiente de variação (CV) é uma medida de dispersão para estimar a precisão de experimentos. Representa o desvio-padrão residual expresso como porcentagem da média geral do experimento. Sua 
principal função é a comparação de experimentos sem que haja igualdade do número de repetições. Quanto menor o coeficiente de variação maior a precisão do experimento para determinada variável. Os experimentos com baixa precisão aumentam as chances de ocorrência de erro do tipo II em que se observa diferença não significativa entre tratamentos quando essa diferença existe ${ }^{(1)}$. Dessa forma, é importante classificar os coeficientes de variação para que os pesquisadores possam avaliar a precisão de experimentos.

Em experimentação com coelhos no Brasil, não há um referencial de valores de CV específico que identifique faixas de classificação quanto à magnitude (baixo, médio, alto e muito alto), a exemplo do que ocorre na experimentação com o milho ${ }^{(2)}$, com o citrus ${ }^{(3)}$, com a suinocultura ${ }^{(4)}$, com a bovinocultura de corte ${ }^{(5)}$, com a cultura do arroz de terras altas ${ }^{(6)}$, com gramíneas forrageiras ${ }^{(7)}$, com a cultura da soja ${ }^{(8)}$, com a cultura do meloeiro ${ }^{(9)}$, com a nutrição de equinos ${ }^{(10)}$, com frangos de corte $^{(11)}$, com a umidade do solo em experimentação agrícola ${ }^{(12)}$, com poedeiras comerciais ${ }^{(13)}$ e com a cultura do tomate em ambientes protegidos ${ }^{(14)}$.

Sendo assim, fica evidente a necessidade de se estudar a distribuição dos valores de CVs em pesquisas com coelhos, pois a maioria dos pesquisadores têm comparado os seus resultados com aqueles propostos por Gomes ${ }^{(1)}$, em que são considerados os CVs baixos com valores inferiores a $10 \%$, médios entre 10 e $20 \%$, altos entre 20 e $30 \%$ e muito altos se superiores a $30 \%$.

Amaral et al. ${ }^{(3)}$ e Judice et al. ${ }^{(4)}$ sugeriram verificar a normalidade da distribuição dos CVs para encontrar as faixas de variabilidade, que se baseiam na média e no desvio-padrão. Entretanto, Costa et al. ${ }^{(6)}$ sugeriram um método alternativo de classificação dos CVs que pode ser aplicado independentemente da distribuição de probabilidade dos valores de CV. Este método baseou-se no uso da mediana (MD) e do pseudo-sigma (PS), medidas estas, segundo o autor, mais resistentes que a média e o desvio-padrão.

O objetivo deste trabalho foi elaborar faixas específicas de classificação dos CVs das principais variáveis utilizadas na experimentação com coelhos no Brasil, utilizando-se a metodologia proposta por Costa et al. ${ }^{(6)}$.

\section{Material e Métodos}

Os valores dos CVs foram obtidos dos trabalhos selecionados por meio de uma revisão sistemática das pesquisas brasileiras em cunicultura entre 1996 e 2010. Utilizaram-se como critérios de inclusão apenas artigos científicos na área de alimentação e nutrição de coelhos entre 2000 e 2010.

Os 34 estudos que atingiram os critérios de inclusão, utilizados para a execução deste trabalho, foram Arruda et al. ${ }^{(15)}$; Arruda et al. ${ }^{(16)}$; Dias et al. ${ }^{(17)}$; Faria et al. ${ }^{(18)}$; Santiago et al. ${ }^{(19)}$; Scapinello et al. ${ }^{(20)}$; Valente et al. ${ }^{(21)}$; Scapinello et al. ${ }^{(22)}$; Scapinello et al. ${ }^{(23)}$; Arruda et al. ${ }^{(24)}$; Cavalcante et al. ${ }^{(25)}$; Michelan et al. ${ }^{(26)}$; Arruda et al. ${ }^{(27)}$; Arruda et al. ${ }^{(28)}$; Furlan et al. ${ }^{(29)}$; Furlan et al. ${ }^{(30)}$; Vieira et al. ${ }^{(31)}$; Faria et al. ${ }^{(32)}$; Furlan et al. ${ }^{(33)}$; Santos et al. ${ }^{(34)}$; Furlan et al. ${ }^{(35)}$; Lui et al. ${ }^{(36)}$; Lui et al. ${ }^{(37)}$; Ferreira et al. ${ }^{(38)}$; Furlan et al. ${ }^{(39)}$; Furlan et al. ${ }^{(40)}$; Michelan et al. ${ }^{(41)}$; Scapinello et al. ${ }^{(42)}$; Barbosa et al. ${ }^{(43)}$; Dávila et al. ${ }^{(44)}$; Ferreira et al. ${ }^{(45)}$; Faria et al. ${ }^{(46)}$; Haponik et al. ${ }^{(47)}$; Euler et al. ${ }^{(48)}$.

Foram coletados os CVs das variáveis: consumo de ração $(\mathrm{CR})$, peso vivo (PV), ganho de peso diário (GPD), conversão alimentar (CA), peso da carcaça (PC), rendimento de carcaça (RC), coeficiente de digestibilidade aparente da matéria seca (CDMS), coeficiente de digestibilidade aparente da proteína bruta (CDPB), coeficiente de digestibilidade aparente da energia bruta (CDEB), coeficiente de digestibilidade aparente da matéria orgânica (CDMO), coeficiente de digestibilidade aparente da fibra em detergente ácido (CDFDA), coeficiente de digestibilidade aparente da fibra em detergente neutro (CDFDN), coeficiente de digestibilidade aparente do amido (CDA) e coeficiente de digestibilidade aparente do extrato etéreo (CDEE). Os CVs de todas as variáveis foram expressos em porcentagem (\%).

Calculou-se o número de observações, o valor mínimo, o valor máximo, a média e o desvio padrão. A mediana $(\mathrm{MD})$ foi calculada pela fórmula: $\mathrm{MD}=(\mathrm{Q} 1+\mathrm{Q} 3) / 2$, em que $\mathrm{Q} 1$ é o primeiro quartil e Q3 é o terceiro quartil. Calculou-se o pseudo-sigma (PS) por meio da fórmula: $\mathrm{PS}=(\mathrm{Q} 3-\mathrm{Q} 1) / 1,35$. A normalidade dos dados foi verificada por meio do teste de Cramer-Von-Mises. Foram elaborados 
intervalos dos CVs segundo a metodologia proposta por Costa et al.(6) em função da MD e do PS. As faixas de classificação dos CVs foram determinadas da seguinte maneira: baixo (CV $\leq \mathrm{MD}-\mathrm{PS})$, médio (MD $-\mathrm{PS}<\mathrm{CV} \leq \mathrm{MD}+\mathrm{PS})$, alto (MD $+\mathrm{PS}<\mathrm{CV} \leq \mathrm{MD}+2 \mathrm{PS})$ e muito alto (CV $>\mathrm{MD}+2 \mathrm{PS})$. O PS é o desvio-padrão que uma distribuição normal precisaria ter a fim de produzir a mesma amplitude interquartílica com os dados utilizados. Essa interpretação do PS é justificada pela presença do valor 1,35 no seu cálculo que é obtido a partir da distribuição normal correspondente à distância entre Q1 e Q3, que equivale a $50 \%$ dos dados, deixando $25 \%$ em cada extremidade ${ }^{(6)}$. Quando os dados não têm distribuição normal o uso do PS como medida de dispersão será mais resistente que o desvio-padrão(6).

\section{Resultados e Discussão}

O teste de normalidade de Cramer-Von-Mises mostrou que somente os CVs das variáveis GPD, CA, RC, CDMS, CDEB, CDMO, CDFDA, CDFDN apresentaram distribuição normal com 5\% de significância. Por isso, foi adotada na presente análise a metodologia para classificação de CV proposta por Costa et al. ${ }^{(6)}$ que não pressupõe a normalidade dos dados. Na Tabela 1 , encontram-se os resultados das estatísticas descritivas obtidas a partir dos valores de $\mathrm{CV}$ encontrados na literatura e o teste de normalidade.

Tabela 1. Número de observações (N), valor mínimo (Min), valor máximo (Max), média $(\mathrm{X})$, desvio padrão (Dp), mediana, pseudo-sigma (PS) e teste de Cramer-Von Mises para normalidade dos coeficientes de variação de ração (CR), peso vivo (PV), ganho de peso diário (GPD), conversão alimentar (CA), peso da carcaça (PC), rendimento de carcaça (RC), coeficiente de digestibilidade aparente da matéria seca (CDMS), coeficiente de digestibilidade aparente da proteína bruta (CDPB), coeficiente de digestibilidade aparente da energia bruta (CDEB), coeficiente de digestibilidade aparente da matéria orgânica (CDMO), coeficiente de digestibilidade aparente da fibra em detergente ácido (CDFDA), coeficiente de digestibilidade aparente da fibra em detergente neutro (CDFDN), coeficiente de digestibilidade aparente do amido (CDA), coeficiente de digestibilidade aparente do extrato etéreo (CDEE) em coelhos

\begin{tabular}{lcccccccc}
\hline Variável & $\mathbf{N}$ & Min & Max & $\mathbf{X}$ & $\mathbf{D p}$ & Mediana & PS & Normalidade \\
\hline CR & 36 & 3,16 & 15,64 & 8,66 & 3,41 & 8,09 & 4,53 & $>0,05$ \\
PV & 21 & 3,07 & 19,36 & 7,73 & 3,57 & 8,00 & 2,56 & $>0,05$ \\
GPD & 37 & 2,37 & 29,83 & 12,01 & 5,71 & 10,75 & 4,77 & $<0,05$ \\
CA & 37 & 0,01 & 26,5 & 10,83 & 5,83 & 9,70 & 5,73 & $<0,05$ \\
PC & 17 & 4,89 & 22,54 & 9,77 & 4,46 & 8,62 & 3,50 & $>0,05$ \\
RC & 23 & 2,03 & 11,06 & 4,10 & 1,77 & 3,85 & 0,86 & $<0,05$ \\
CDMS & 22 & 2,21 & 24,06 & 6,86 & 5,16 & 5,45 & 4,68 & $<0,05$ \\
CDPB & 19 & 1,01 & 13,89 & 6,48 & 4,25 & 5,37 & 6,01 & $>0,05$ \\
CDEB & 21 & 1,73 & 26,06 & 7,75 & 6,80 & 5,03 & 3,88 & $<0,05$ \\
CDMO & 13 & 0,95 & 14,89 & 4,65 & 3,89 & 2,77 & 2,33 & $<0,05$ \\
CDFDA & 9 & 2,40 & 26,57 & 12,99 & 6,76 & 13,16 & 4,66 & $>0,05$ \\
CDFDN & 13 & 1,52 & 29,57 & 13,41 & 8,45 & 10,03 & 6,88 & $<0,05$ \\
CDA & 7 & 0,33 & 3,91 & 1,42 & 1,22 & 1,19 & 3,30 & $<0,05$ \\
CDEE & 6 & 1,78 & 23,72 & 7,80 & 8,07 & 5,42 & 1,01 & $>0,05$ \\
\hline
\end{tabular}


Na literatura consultada, foi obtido maior número de CVs para as variáveis GPD, CA e CR com valores 37, 37 e 36, respectivamente. As medianas dos CVs situaram-se entre 1,19 e 13,16. As variáveis que apresentaram maior variabilidade foram CDFDN, CDPB e CA com valores do pseudo-sigma de 6,88, 6,01 e 5,73, respectivamente. A variável com menor variabilidade foi à $\mathrm{RC}$, apresentando pseudosigma de 0,86. Com base nos valores de máximo e mínimo, notou-se grande amplitude dos dados dentro das variáveis. Isso demonstra influência de grande número de fatores e justifica a necessidade de classificação específica dos coeficientes de variação referente a cada resposta observada.

Na Tabela 2 são apresentadas as faixas de classificação dos CVs para as variáveis estudadas, utilizandose a metodologia proposta por Costa et al. ${ }^{(6)}$.

Verifica-se que a classificação dos CVs deste trabalho difere da classificação de Gomes ${ }^{(1)}$, geralmente utilizada em trabalhos científicos que usam limites fixos, qualquer que seja a variável analisada ${ }^{(1)}$. Por isso, a classificação deste trabalho pode ser utilizada como referência pelos pesquisadores para verificarem se os resultados dos CVs obtidos estão, ou não, dentro de uma faixa esperada de valores na experimentação com coelhos no Brasil.

Como pode ser observado na Tabela 2, cada variável apresentou faixa de valores de CV específica, o que ressalta a necessidade de abordagem também distinta dessa medida de variação, conforme a natureza dos dados. Por exemplo, na classificação normalmente utilizada ${ }^{(1)}$, um $\mathrm{CV}<10 \%$ é tido como baixo, sendo que nesta pesquisa para CR, GPD, CA, PC, CDMS, CDPB, CDFDA e CDFDN este CV é considerado como médio, para as variáveis PV, CDEB e CDEE este CV é considerado como alto e para as demais variáveis analisadas CV de $10 \%$ é classificado como muito alto.

Tabela 2. Faixas de coeficiente de variação $(\mathrm{CV}, \%)$ para consumo de ração $(\mathrm{CR})$, peso vivo $(\mathrm{PV})$, ganho de peso diário (GPD), conversão alimentar (CA), peso da carcaça (PC), rendimento de carcaça $(\mathrm{RC})$, coeficiente de digestibilidade aparente da matéria seca (CDMS), coeficiente de digestibilidade aparente da proteína bruta (CDPB), coeficiente de digestibilidade aparente da energia bruta (CDEB), coeficiente de digestibilidade aparente da matéria orgânica (CDMO), coeficiente de digestibilidade aparente da fibra em detergente ácido (CDFDA), coeficiente de digestibilidade aparente da fibra em detergente neutro (CDFDN), coeficiente de digestibilidade aparente do amido (CDA), coeficiente de digestibilidade aparente do extrato etéreo (CDEE) em coelhos

\begin{tabular}{lcccc}
\hline Variável & Baixo & Médio & Alto & Muito Alto \\
\hline $\mathrm{CR}$ & $\mathrm{CV} \leq 4,43$ & $4,43<\mathrm{CV} \leq 13,48$ & $13,48<\mathrm{CV} \leq 18,01$ & $\mathrm{CV}>18,01$ \\
$\mathrm{PV}$ & $\mathrm{CV} \leq 4,55$ & $4,55<\mathrm{CV} \leq 9,67$ & $9,67<\mathrm{CV} \leq 12,24$ & $\mathrm{CV}>12,24$ \\
$\mathrm{GPD}$ & $\mathrm{CV} \leq 6,51$ & $6,51<\mathrm{CV} \leq 16,05$ & $16,05<\mathrm{CV} \leq 20,82$ & $\mathrm{CV}>20,82$ \\
$\mathrm{CA}$ & $\mathrm{CV} \leq 5,33$ & $5,33<\mathrm{CV} \leq 16,79$ & $16,79<\mathrm{CV} \leq 22,53$ & $\mathrm{CV}>22,53$ \\
$\mathrm{PC}$ & $\mathrm{CV} \leq 5,18$ & $5,18<\mathrm{CV} \leq 12,19$ & $12,19<\mathrm{CV} \leq 15,69$ & $\mathrm{CV}>15,69$ \\
$\mathrm{RC}$ & $\mathrm{CV} \leq 2,99$ & $2,99<\mathrm{CV} \leq 4,71$ & $4,71<\mathrm{CV} \leq 5,57$ & $\mathrm{CV}>5,57$ \\
$\mathrm{CDMS}$ & $\mathrm{CV} \leq 1,91$ & $1,91<\mathrm{CV} \leq 11,27$ & $11,27<\mathrm{CV} \leq 15,95$ & $\mathrm{CV}>15,95$ \\
$\mathrm{CDPB}$ & $\mathrm{CV} \leq 0,57$ & $0,57<\mathrm{CV} \leq 12,58$ & $12,58<\mathrm{CV} \leq 18,59$ & $\mathrm{CV}>18,59$ \\
$\mathrm{CDEB}$ & $\mathrm{CV} \leq 1,67$ & $1,67<\mathrm{CV} \leq 9,43$ & $9,43<\mathrm{CV} \leq 13,31$ & $\mathrm{CV}>13,31$ \\
$\mathrm{CDMO}$ & $\mathrm{CV} \leq 1,66$ & $1,66<\mathrm{CV} \leq 6,33$ & $6,33<\mathrm{CV} \leq 8,66$ & $\mathrm{CV}>8,66$ \\
$\mathrm{CDFDA}$ & $\mathrm{CV} \leq 7,80$ & $7,80<\mathrm{CV} \leq 17,11$ & $17,11<\mathrm{CV} \leq 21,77$ & $\mathrm{CV}>21,77$ \\
$\mathrm{CDFDN}$ & $\mathrm{CV} \leq 5,58$ & $5,58<\mathrm{CV} \leq 19,35$ & $19,35<\mathrm{CV} \leq 26,23$ & $\mathrm{CV}>26,23$ \\
$\mathrm{CDA}$ & $\mathrm{CV} \leq 1,94$ & $1,94<\mathrm{CV} \leq 8,84$ & $8,84<\mathrm{CV} \leq 11,85$ & $\mathrm{CV}>11,85$ \\
$\mathrm{CDEE}$ & $\mathrm{CV} \leq 0,22$ & $0,22<\mathrm{CV} \leq 2,25$ & $2,25<\mathrm{CV} \leq 3,26$ & $\mathrm{CV}>3,26$ \\
\hline
\end{tabular}


A variável RC destaca-se por apresentar a faixa de classificação mais estreita, revelando-se ser uma variável bastante estável, seguidas das variáveis CDA, CDMO e PV, respectivamente. Ao contrário destas variáveis, o CDFDN, CDPB, CA e GPD apresentam as maiores faixas de classificação dos CV.

As variáveis CDFDN e CDPB apresentam maior variação na faixa de classificação, provavelmente, devido aos diferentes métodos de obtenção desses coeficientes, uma vez que estes estão susceptíveis a erros na mensuração da resposta. Com relação à CA e ao GPD, essa variação deve estar relacionada com as condições distintas nas quais foram realizados os experimentos.

Sendo assim, fica evidente com os resultados obtidos, a necessidade de classificação específica do CV para as variáveis estudadas em experimentação com coelhos, como ocorre na área agronômica $(2,7-9,12,14,49,50)$ e zootécnica ${ }^{(4,5,10,11,13)}$, permitindo ao pesquisador verificar a precisão das suas pesquisas e também dos trabalhos publicados na literatura.

\section{Conclusão}

Os resultados encontrados permitem concluir que, para cada variável utilizada em experimentação na cunicultura, existe uma distribuição específica de valores de coeficiente de variação que podem ser utilizadas como referência para determinar a precisão experimental. As faixas de classificação encontradas para cunicultura são diferentes daquelas para outras espécies animais e das utilizadas em experimentação agronômica.

\section{Referências}

1. Gomes FP. Curso de estatística experimental. 13 ed. São Paulo: Nobel; 2000. 451p. Português.

2. Scapim CA, Carvalho CGP, Cruz CD. Uma proposta de classificação dos coeficientes de variação para a cultura do milho. Pesquisa Agropecuária Brasileira. 1995;30(5):683-686.

3. Amaral AM, Muniz JA, Souza M. Avaliação do coeficiente de variação como medida da precisão na experimentação com citros. Pesquisa Agropecuária Brasileira, 1997;32 (12):1221-1225.

4. Judice MG, Muniz JA, Carvalheiro R. Avaliação do coeficiente de variação na experimentação com suínos. Ciência e Agrotecnologia. 1999;23(1):170-173.

5. Judice MG; Muniz JA, Aquino LH, Bearzoti E. Avaliação da precisão experimental em ensaios com bovinos de corte. Ciência e Agrotecnologia. 2002;26(5):1035-1040.

6. Costa NHAD, Seraphin JC, Zimmermann FJP. Novo método de classificação de coeficientes de variação para a cultura do arroz de terras altas. Pesquisa Agropecuária Brasileira. 2001;37(6):243-249.

7. Clemente AL, Muniz JA, Avaliação do coeficiente de variação em experimentos com gramíneas forrageiras. Ciência e Agrotecnologia. 2002;26:197-203.

8. Carvalho CGP, Arias CAA, Toledo JFF, Almeida LA, Kiihl RAS, Oliveira MF, Hiromoto DM, Takeda C. Proposta de classificação dos coeficientes de variação em relação à produtividade e altura da planta de soja. Pesquisa Agropecuária Brasileira. 2003;38:187-193.

9. Lima LL, Nunes GHS, Bezerra Neto F. Coeficientes de variação de algumas características do meloeiro: uma proposta de classificação. Horticultura Brasileira, 2004;22:14-17.

10. Lana AMQ, Soares Neto J, Almeida FQ, Rezende ASC, Prates RC. Classificação de coeficientes de variação na experimentação com nutrição de eqüinos. Arquivo Brasileiro de Medicina Veterinária e Zootecnia. 2006;58(5):854-859. 
11. Mohallem DF, Tavares M, Silva PL, Guimarães EC, Freitas RF. Avaliação do coeficiente de variação com medida de precisão em experimentos com frangos de corte. Arquivo Brasileiro de Medicina Veterinária e Zootecnia. 2008;60(2):449-453.

12. Costa FM, Oliveira JM, Guimarães EC, Tavares M. Classificação do coeficiente de variação da umidade do solo em experimentação agrícola. Famat R [Internet]. 2008 Feb [cited 2011 Nov 12];10. Available from: $<$ http://www.portal.famat.ufu.br/sites/famat.ufu.br/files/Anexos/Bookpage/Famat revista_artigo_04.pdf $>$

13. Faria Filho DE, Dias AN, Veloso ALC, Bueno CFD, Couto FAP, Matos Júnior JB, Barreto KZO, Rodrigues PA, Carneiro WA. Classification of coefficients of variation in experiments with commercial layers. Brazilian Journal of Poultry Science. 2010;12(4): 215-217.

14. Cruz EA, Moreira GR, Paula MO, Oliveira ACM. Coeficiente de variação como medida de precisão em experimentos com tomate em ambiente protegido. Centro Científico Conhecer. 2012;8(14):220-233.

15. Arruda AMV, Carregal RD, Ferreira RG. Desempenho produtivo e atividade microbiana cecal de coelhos alimentados com dietas contendo diferentes níveis de amido. Revista Brasileira de Zootecnia. 2000a;29(3):762768.

16. Arruda AMV, Carregal RD, Ferreira RG. Digestibilidade aparente de dietas contendo diferentes níveis de amido para coelhos em crescimento. Revista Brasileira de Zootecnia. 2000b;29(3):769-775.

17. Dias JCCA, Ferreira WM, Santiago GS, Valente SS, Colares FAP. Níveis decrescentes de proteína em dietas suplementadas com complexo enzimático para coelhos em crescimento. 1. Desempenho produtivo. Arquivo Brasileiro de Medicina Veterinária e Zootecnia. 2000;52(2):160-166.

18. Faria HG, Scapinello C, Furlan AC, Moreira I, Martins EN. Valor Nutritivo das leveduras de recuperação (Saccharomycessp), seca por rolo rotativo ou por "spray-dry", para coelhos em crescimento. Revista Brasileira de Zootecnia. 2000;29(6):1750-1753.

19. Santiago GS, Dias JCCA, Ferreira WM, Valente SS. Níveis decrescentes de proteína em dietas suplementadas com complexo enzimático, para coelhos em crescimento. 2. Parâmetros digestivos e composição química do conteúdo cecal. Arquivo Brasileiro de Medicina Veterinária e Zootecnia. 2000;52(2):167-172.

20. Scapinello C, Furlan AC, Jobim CC, Faria HG, Figueiredo DF, Hernandes AB. Valor nutritivo e utilização do feno de leucena (Leucaenaleucocephala cv. Cunningham) para coelhos em crescimento. Acta Scientiarum, Animal Sciences. 2000b;22(3):829-833.

21.Valente SS, Santiago GS, Ferreira WM, Dias JCCA. Desempenho de coelhos em crescimento recebendo dietas com suplementação enzimática. Arquivo Brasileiro de Medicina Veterinária e Zootecnia. 2000;52(2):173177.

22. Scapinello C, Faria HG, Furlan AC, Michelan AC. Efeito da utilização de oligossacarídeo manose e acidificantes sobre o desempenho de coelhos em crescimento. Revista Brasileira de Zootecnia. 2001a;30(4):12721277.

23. Scapinello C, Faria HG, Furlan AC, Michelan AC, Santolin MLR. Efeito do uso de oligossacarídeo manose e acidificantes em rações com alto teor de amido, para coelhos em crescimento. Acta Scientiarum, Animal Sciences. 2001b;23(4):1039-1043.

24. Arruda AMV, Lopes DC, Ferreira WM, Rostagno HS, Queiroz AC, Pereira ES, Albino LFT, Silva JF. Digestibilidade aparente dos nutrientes de rações contendo diferentes fontes de fibra e níveis de amido com coelhos em crescimento. Revista Brasileira de Zootecnia. 2002;31(3):1166-1175.

25. Cavalcante SG, Ferreira WM, Valente SS, Santiago GS, Dias JCCA, Naranjo AP. Biodisponibilidade de cobre de diferentes fontes para coelhos. Arquivo Brasileiro de Medicina Veterinária e Zootecnia. 2002;54(3): 290-294.

26. Michelan AC, Scapinello C, Natali MRM, Furlan AC, Sakaguti ES, Faria HG, Santolin MLR, Hernandes 
AB. Utilização de probiótico, ácido orgânico e antibiótico em dietas para coelhos em crescimento: ensaio de digestibilidade, avaliação da morfometria intestinal e desempenho. Revista Brasileira de Zootecnia. 2002;31(6): 2227-2237.

27. Arruda AMV, Lopes DC, Ferreira WM, Rostagno HS, Queiroz AC, Pereira ES, Ferreira AS, Silva JF. Desempenho e características de carcaça de coelhos alimentados com rações contendo diferentes níveis de amido e fontes de fibra. Revista Brasileira de Zootecnia. 2003a;32(6):1311-1320.

28. Arruda AMV, Lopes DC, Ferreira WM, Rostagno HS, Queiroz AC, Pereira ES, Silva JF, Jham GN. Atividade microbiana cecal e contribuição nutricional da cecotrofia em coelhos alimentados com rações contendo diferentes fontes de fibra e níveis de amido. Revista Brasileira de Zootecnia. 2003b;32(4):891-902.

29. Furlan AC, Monteiro RT, Scapinello C, Moreira I, Murakami AE, Otutumi LK, Santolin MLR. Valor nutritivo e desempenho de coelhos em crescimento alimentados com rações contendo milho extrusado. Revista Brasileira de Zootecnia. 2003a;32(5):1157-1165.

30. Furlan AC, Scapinello C, Toral FLB, Faria HG, Moreira I, Murakami AE, Santolin MLR. Valor nutritivo e desempenho de coelhos alimentados com rações contendo milheto (Pennisetum glaucum( L.) R.Br). Revista Brasileira de Zootecnia. 2003b;32(1):123-131.

31. Vieira FS, Gomes AVC, Pessoa MF. Efeito da granulometria do bagaço de cana sobre as características digestivas e a contribuição nutritiva dos cecotrofos. Revista Brasileira de Zootecnia. 2003;32(4):935-941.

32. Faria HG, Scapinello C, Peralta RM, Gidenne T, Furlan AC, Andreazzi MA. Digestibilidade e desempenho de coelhos oriundos de quatro padrões de alimentação até a desmama alimentados com dietas contendo diferentes níveis de amido após a desmama. Revista Brasileira de Zootecnia. 2004b;33(5):1172-1180.

33. Furlan AC, Monteiro RT, Scapinello C, Moreira I, Murakami AE, Martins EN. Avaliação nutricional do triticale extrusado ou não para coelhos em crescimento. Acta Scientiarum, Animal Sciences. 2004;26(1):49-55.

34. Santos EA, Lui JF, Scapinello C. Efeito dos níveis de fibra em detergente ácido sobre os coeficientes de digestibilidade das dietas e desempenho de coelhos em crescimento. Acta Scientiarum, Animal Sciences. 2004;26(1):79-86.

35. Furlan AC, Scapinello C, Moreira I, Murakami AE, Santolin MLR, Otutumi LK. Avaliação nutricional da raspa integral de mandioca extrusada ou não para coelhos em crescimento. Acta Scientiarum, Animal Sciences. 2005;27(1):99-103.

36. Lui JF, Andrade BRP, Oliveira MC, Santos EA, Caires DR. Valor nutritivo do feno de alfafa e do pé de milho moído para coelhos em crescimento. ARS Veterinaria. 2005a;21(supl.):142-146.

37. Lui JF, Oliveira MC, Caires DR, Cancherini LC. Desempenho, rendimento de carcaça e pH cecal de coelhos em crescimento alimentados com dietas contendo níveis de probiótico. Ciência Animal Brasileira. 2005b;6(2):87-93.

38. Ferreira VPA, Ferreira WM, Saliba EOS, Scapinello C, Teixeira AO, Kamwa EB. Digestibilidade, cecotrofia, desempenho e rendimento de carcaça de coelhos em crescimento alimentados com rações contendo óleo vegetal ou gordura animal. Revista Brasileira de Zootecnia. 2006;35(4):1696-1704.

39. Furlan AC, Santolin MLR, Scapinello C, Moreira I, Faria HG. Avaliação nutricional do trigo mourisco (Fagopyrumesculentum, Moench) para coelhos em crescimento. Acta Scientiarum, Animal Sciences. 2006a;28(1):21-26.

40. Furlan AC, Scapinello C, Moreira I, Martins EN. Avaliação nutricional da silagem de grãos úmidos de sorgo de baixo ou de alto conteúdo de tanino para coelhos em crescimento. Revista Brasileira de Zootecnia. 2006b;35(3):775-784.

41. Michelan AC, Scapinello C, Furlan AC, Martins EM, Faria HG, Andreazzi MA. Utilização da casca de mandioca desidratada na alimentação de coelhos. Acta Scientiarum, Animal Sciences. 2006;28(1):31-37. 
42. Scapinello C, Michelan AC, Furlan AC, Martins EM, Faria HG, Andreazzi MA. Utilização da farinha de varredura de mandioca na alimentação de coelhos. Acta Scientiarum, Animal Sciences. 2006;28(1):39-45.

43. Barbosa JG, Silva LPG, Oliveira EM, Pereira WE, Cavalcante Neto A, Oliveira MRT, Medeiros NA, Motas JKM. Efeitos da inclusão da levedura seca (Saccharomycescerevisiae) sobre a carcaça e na composição da carne de coelhos. Ciência Animal Brasileira. 2007;8(1):51-58.

44. Dávila NFP, Gomes AVC, Pessôa MF, Crespi MPL, Coll JFC. Substituição do farelo de soja por farelo de algodão na alimentação de coelhos em crescimento. Acta Scientiarum, Animal Sciences. 2007;29(3):277-282.

45. Ferreira WM, Herrera ADPN, Scapinello C, Fontes DO, Machado LC, Ferreira SRA. Digestibilidade aparente dos nutrientes de dietas simplificadas baseadas em forragens para coelhos em crescimento. Arquivo Brasileiro de Medicina Veterinária e Zootecnia. 2007;59(2):451-458.

46. Faria HG, Ferreira WM, Scapinello C, Oliveira CEA. Efeito da utilização de dietas simplificadas, à base de forragem, sobre a digestibilidade e o desempenho de coelhos Nova Zelândia. Revista Brasileira de Zootecnia. 2008;37(10):1797-1801.

47. Haponik CAV, Espíndola GB, Freitas ER, Raquel DL, Ramos LO, Chaves CS. Avaliação nutricional de dietas contendo farelo de coco fornecido a coelhos destinados ao abate. Acta Scientiarum, Animal Sciences. 2009;31(4):357-364.

48. Euler ACC, Ferreira WM, Teixeira EA, Lana AMQ, Guedes RMC, Avelar AC. Desempenho, digestibilidade e morfometria da vilosidade ileal de coelhos alimentados com níveis de inclusão de "Lithothamnium". Revista Brasileira de Saúde e Produção Animal. 2010;11(1):91-103.

49. Cargnelutti Filho A, Storck L. Estatísticas de avaliação da precisão experimental em ensaios de cultivares de milho. Pesquisa Agropecuária Brasileira. 2007;42(1):17-24.

50. Oliveira RL, Muniz JA, Andrade MJB, Reis RL. Precisão experimental em ensaios com a cultura do feijão. Ciência e Agrotecnologia. 2009;33(1):113-119. 